\title{
Technique of Coaxial Frame in Reflection for the Characterization of Single and Multilayer Materials with Correction of Air Gap
}

\author{
Zarral Lamia, ${ }^{1}$ Djahli Farid, ${ }^{1}$ and Ndagijimana Fabien ${ }^{2}$ \\ ${ }^{1}$ Laboratory of Scientific Instrumentation Institute of Electronic, FERHAT Abbess University, 19000 Setif, Algeria \\ ${ }^{2}$ Laboratory of IMEP-LAHC Minatec, Institute of Microelectronics, Electromagnetism and Photonics, \\ 38000 Grenoble, France
}

Correspondence should be addressed to Zarral Lamia; lamia_zarral@yahoo.fr

Received 6 March 2014; Revised 2 June 2014; Accepted 17 June 2014; Published 24 July 2014

Academic Editor: Wenhua Yu

Copyright (C) 2014 Zarral Lamia et al. This is an open access article distributed under the Creative Commons Attribution License, which permits unrestricted use, distribution, and reproduction in any medium, provided the original work is properly cited.

\begin{abstract}
Techniques based on fixture probes in reflection are used in microwave reflectometry as a novel diagnostic tool for detection of skin cancers, for complex permittivity measurements on liquid samples and oil shale, and for complex dielectric permittivity of animals' organs and tissues measurements in microwave band for the needs of modern veterinary medicine. In this work, we have developed a technique to characterize multilayer materials in a broadband frequency range. A coaxial probe in reflection has been specially developed for microelectronic substrate. Using SMA connector, loss tangent of $10^{-4}$ and relative permittivity have been measured with an error of $0.145 \%$. The extension of the coaxial probe in reflection technique to multilayer substrates such as Delrin and Teflon permitted to measure bilayer material provided the good knowledge of electrical parameters and dimensions of one layer. In the coaxial transmission line method, a factor that greatly influences the accuracy of the results is the air gaps between the material under test and the coaxial test fixture. In this paper, we have discussed the influence of the air gaps (using samples of $0.5 \mathrm{~mm}$ air gaps) and the measures that can be taken to minimize that influence when material is measured. The intrinsic values thus determined have been experimentally verified. We have described the structure of the test fixture, its calibration issues, and the experimental results. Finally, electromagnetism simulations showed that the best results can be obtained.
\end{abstract}

\section{Introduction}

In this paper, we have developed a method using a coaxial fixture that allows the extraction of electrical parameters of substrates by the reflection measurement. This coaxial probe is in contact at its end with the samples and with a short circuit for calibration. For validation we have measured the Teflon and Delrin (Pome). The validation of the method is first made on monolayer materials and secondly on multilayers substrates. We reversed the positions of the couple of materials (Delrin, Teflon) then (Teflon-Delrin) to check their asymmetries. In the multilayer measurements we use Teflon as reference material.

\section{Principle of the Method}

Figure 1 shows the structure of the line with sample as well as the reference plans of measurements in this method. Our method requires six measures to be made: in a first step we perform one measure of the S11 parameters in open circuit and another one in short circuit for calibration. In a second step we measure the S11 parameters of line without sample, terminated with open circuit and with short circuit at the end. In the third and last step, we measure the S11 parameters of the line with sample terminated with short circuit and with open circuit. 


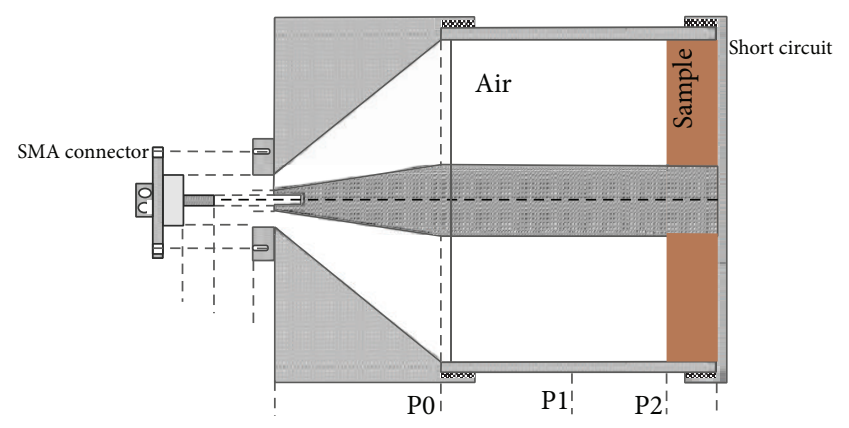

FIGURE 1: Presentation of the coaxial reflective frame with sample.

To move from plan P1 to plan P2, as shown in Figure 1, we apply the following formula [1], with 1 being the length between $\mathrm{P} 0$ and $\mathrm{P} 1$ :

$$
S_{i j}^{\prime}=S_{i j} \cdot e^{2 \cdot j \cdot \gamma l}
$$

\section{Presentation of the Coaxial Frame in Reflection}

A coaxial frame is a coaxial line comprising a central conductor and a cylindrical outer aluminum conductor. This line is connected to the tester by means of an SMA connector, which is placed on one of its ends so as to measure the S11 parameters in which the electrical parameters of the material are extracted [2]. On the termination of the coaxial line we connect a short circuit or open circuit so that the material under test is in direct contact with the short circuit (Figure 1).

For the modeling of this frame we have calculated all the parameters of the transmission line, as well as the dimensions of the samples to be characterized before proceeding to the simulation step.

\section{Electromagnetic Simulations and Validation}

We simulated the structure of the frame with and without sample in the case of loss and lossless dielectric using CST and HFSS11.1 simulators. The simulation is made with the following steps.

4.1. Impedance Matching Bandwidth. The test structure comprises a radiating coaxial transmission line in a semi-infinite environment with a given thickness and ended by a metal plate for short circuit. The ratio of the inner diameter $(2 a)$ and of the outer conductor $(2 b)$ is set so that the characteristic impedance of the transmission line is equal to $50 \Omega$ in without sample.

4.2. Extraction of the Electrical Parameters of the Dielectric. The $S 11$ parameters, obtained by simulation, allow easy access to propagation phase $\gamma l$ in the presence and in the absence of material. Consider

$$
Z(S)=Z n \cdot \frac{1+S}{1-S}
$$

where $Z(S)$ is the impedance characteristic function of $S$ parameters, $\gamma$ is the propagation constant, and $l$ is the length of the line.

In the absence of the dielectric material, we have

$$
\begin{aligned}
& Z c_{\text {air }}=\sqrt{Z c_{\text {air }} c c \cdot Z c_{\text {air }} c o} \\
& \gamma l_{\text {air }}=a \tanh \sqrt{\frac{Z c_{\text {air }} c c}{Z c_{\text {air }} c o}}
\end{aligned}
$$

In the presence of the dielectric material

$$
\begin{gathered}
Z c_{\text {mat }}=\sqrt{Z c_{\text {mat }} c c \cdot Z c_{\text {mat }} c o}, \\
\gamma l_{\text {mat }}=a \tanh \sqrt{\frac{Z c_{\text {mat }} c c}{Z c_{\text {mat }} c o}},
\end{gathered}
$$

where $Z c$ is the characteristic impedance of the line.

These two measures of parameter $\gamma l[3,4]$ allow easy access, theoretically, to parameters $\varepsilon_{r}$ and $\tan \delta_{d}$ of the dielectric material.

From the line impedance in short circuit and open circuit, to extract the propagation parameters of a transmission line, we can measure the $S 11$ reflection parameters in two different configurations, namely, the line in short circuit and the line in open circuit [5]. Any transmission line can be represented by its propagation constant $(Z c, \gamma l)$.

The input impedance $Z_{\text {in }}$ of a transmission line loaded by impedance $Z_{R}$ is determined by the following expression:

$$
Z_{\text {in }}=Z c \frac{Z_{R}+Z c \tanh (\gamma l)}{Z c+Z_{R} \tanh (\gamma l)} .
$$

In the case of a short-circuit line $\left(Z_{R}=0\right)$, the impedance seen at the input line is written as

$$
Z_{\text {in }_{c c}}=Z_{c} \cdot \tanh (\gamma l)=Z_{n} \frac{1+\Gamma_{c c}}{1-\Gamma_{c c}}=Z_{c c},
$$

where $Z_{n}$ is the standard resistance that is equal to $50 \mathrm{Ohm}$ and $\Gamma$ is a coefficient of reflexion.

In the case of an open-circuit line $\left(Z_{R}=\infty\right)$, the impedance seen at the input of the line is

$$
Z_{\mathrm{in}_{c o}}=\frac{Z c}{\tanh (\gamma l)}=Z_{n} \frac{1+\Gamma_{c o}}{1-\Gamma_{c o}}=Z_{c o} .
$$

4.3. Extraction of the Complex Relative Permittivity. The complex relative permittivity and the loss angle are obtained from

$$
\begin{gathered}
\varepsilon_{\mathrm{eff}}=\frac{\gamma l_{\mathrm{mat}}+Z c_{\mathrm{air}}}{\gamma l_{\mathrm{air}}+Z c_{\mathrm{mat}}}, \\
\tan \delta=\frac{\gamma l_{\mathrm{mat}} \cdot Z c_{\mathrm{mat}}}{\gamma l_{\mathrm{air}} \cdot Z c_{\mathrm{air}}},
\end{gathered}
$$

where $\varepsilon_{\text {eff }}$ is the effective relative permittivity and $\tan \delta$ is the dielectric losses. 


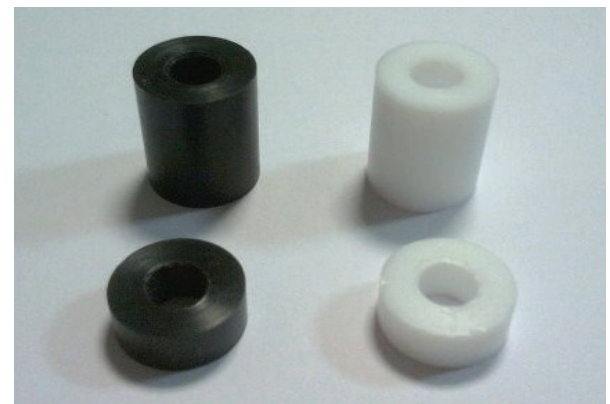

Figure 2: Sample of Delrin and Teflon for $d=5 \mathrm{~mm}$ and $15 \mathrm{~mm}$.

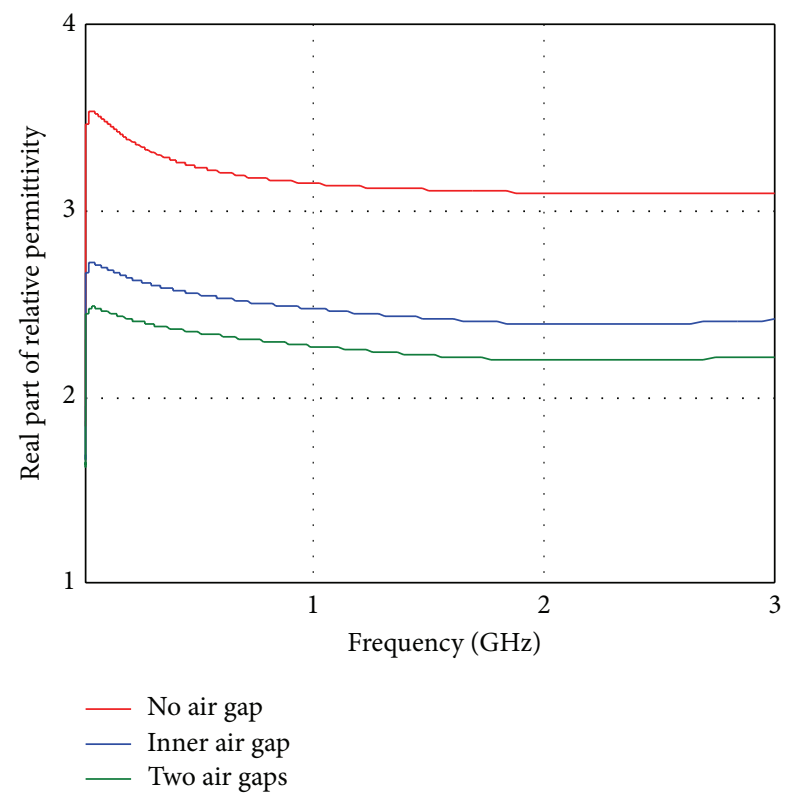

FIGURE 3: Real part of relative permittivity of Delrin.

4.4. Machining of the Samples. The advanced hypothesis of the measurement technique in coaxial line (spread of a single TEM mode in all regions of the line) requires the complete filling of the transverse section of the line by the sample (Figure 2). This involves strong machining constraints of the sample ring because of the presence of an air gap between it and the conductive walls of the line.

Although mechanical tolerances met by the end of the machining process are low, the manual insertion of the sample inside the line inevitably implies the presence of an air gap. It will influence the accuracy of measurement results. To illustrate this problem, we have studied the effect of these errors on the determination of the dielectric permittivity $\varepsilon_{r}$. Errors of measurement due to the presence of air space between the sample and the line conductors are gradually larger as the frequency increases. For this, we have established a correction (sample of $5 \mathrm{~mm}$ long and with no air gap).

4.5. Correction of the Air Gap. In these simulations and to reduce the number of parameters, we have assumed that the air gap between the material and the outer conductor is zero.

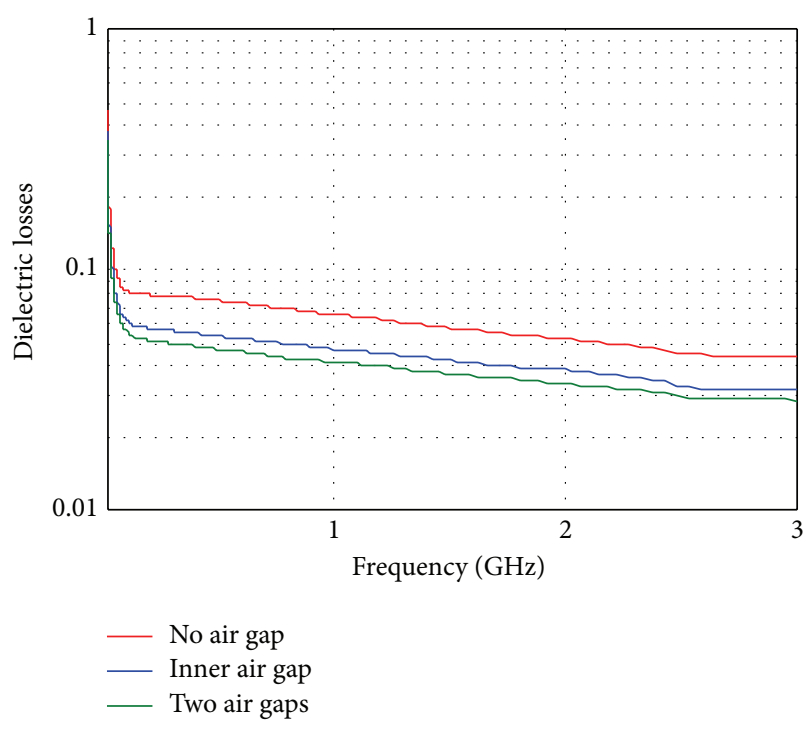

Figure 4: Delrin dielectric losses.

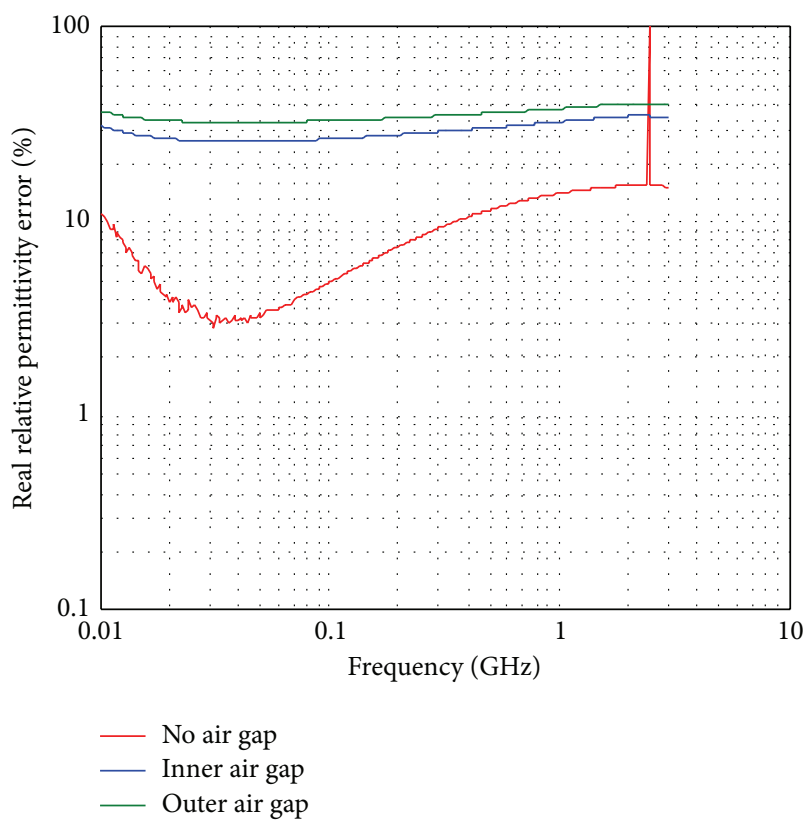

FIGURE 5: Error of relative permittivity of Delrin.

Figure 5 shows that the error increases very rapidly with the thickness of air space and the value of the permittivity of the material. This has the effect of lowering the value of the measured permittivity.

To correct the errors related to the imperfections of the test device (such as the connecting cable of the analyser, the coaxial conical/coaxial transition associated with the discontinuity line/guide, and metal losses due to finite conductivity of the conductors used to make the alignment of the various elements) we added formula (9), in the MathCAD program. A formula has been derived to estimate the effective complex 


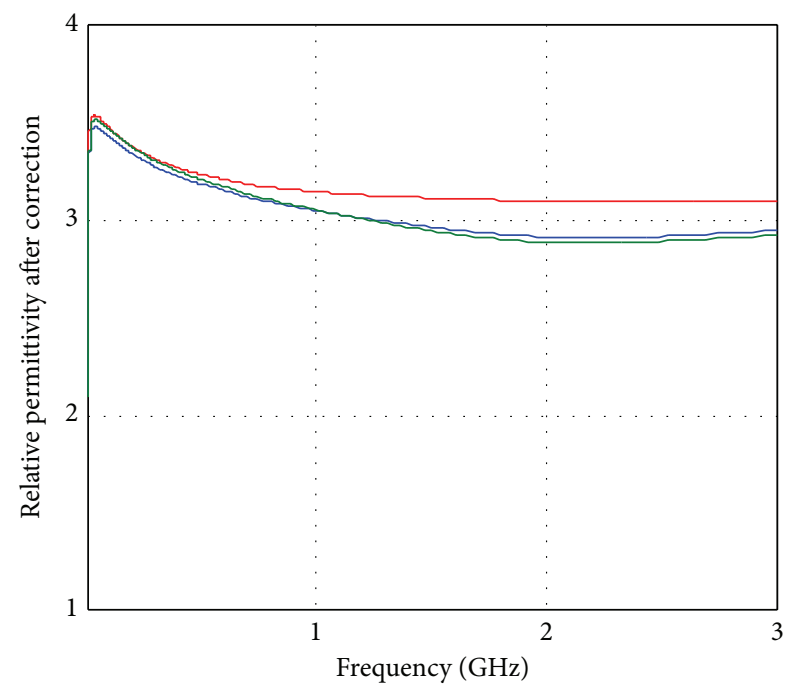

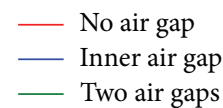

FIGURE 6: Relative permittivity after correction of the Delrin.

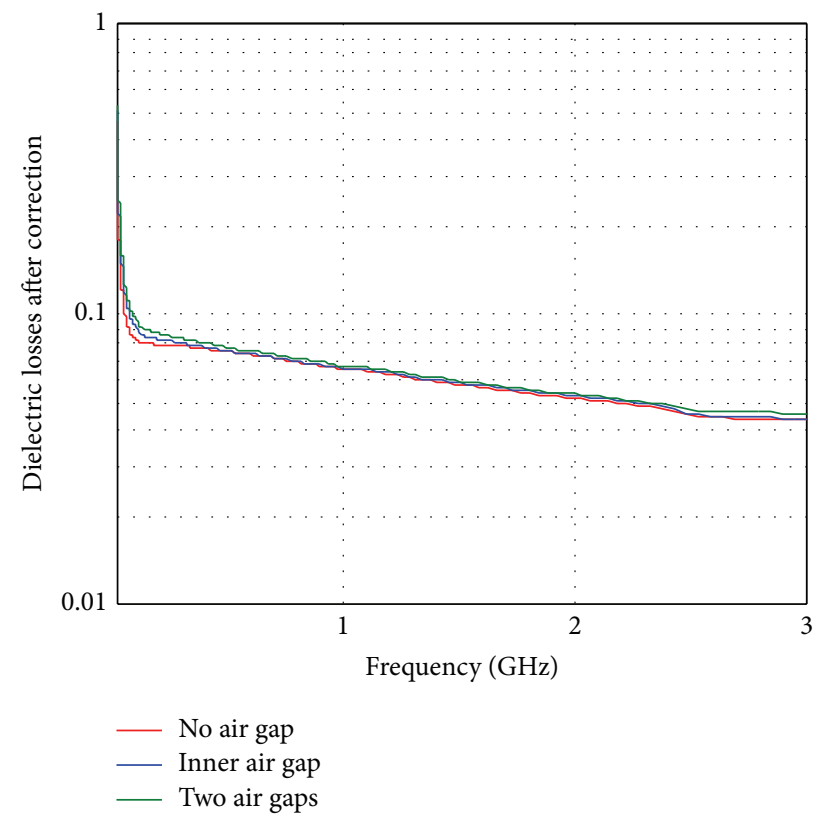

FIgURE 7: Dielectric losses after correction of Delrin.

permittivity $\varepsilon_{r, \text { fff }}^{*}$ from the total capacitance consisting of a mixture of three coaxial layers, air, a bulk material, and air:

$$
\varepsilon_{r, \text { eff }}^{*}=\ln \left(\frac{b}{a}\right)\left[\ln \left(\frac{b / a}{r_{0} / r_{i}}\right)+\frac{\ln \left(r_{0} / r_{i}\right)}{\varepsilon_{r, \text { nom }}^{*}}\right]^{-1},
$$

where $\varepsilon_{r, \text { nom }}^{*}$ is the nominal complex permittivity, $a$ and $b$ are the inner and outer radii of the sample holder, and $r_{i}$ and $r_{0}$ are the inner and outer radii of the sample. To obtain a gap correction formula, this equation can be rearranged by

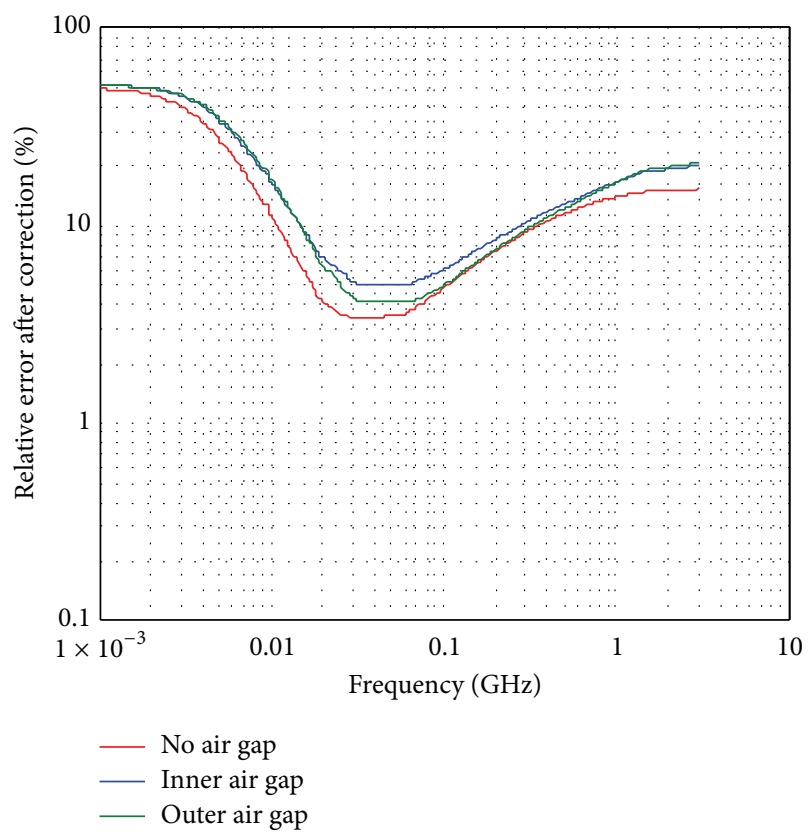

FIGURE 8: Relative permittivity error after correction of Delrin.

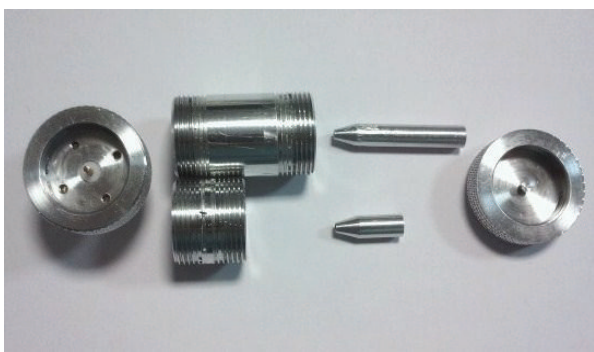

FIGURE 9: Constituents of the small and large coaxial line in reflexion.

renaming $\varepsilon_{r, \text { eff }}^{*} \rightarrow \varepsilon_{r, m}^{*}$ and $\varepsilon_{r, \text { nom }}^{*} \rightarrow \varepsilon_{r, c}^{*}$ (corrected complex permittivity) and solving for $\varepsilon_{r, c}^{*}(11)$.

Wall thickness was calculated by subtracting the inner diameter of the sample from its outer diameter and dividing the difference by 2 .

To extract the initial dielectric loss, we simulate a case with $\left(\sigma_{\mathrm{AP}}\right)$ losses and $\left(\sigma_{\mathrm{SP}}\right)$ lossless dielectrics. The difference between the two extracted electrical conductivities gives the electrical conductivity of the dielectric $\sigma_{d}$ after correction is given by the following:

$$
\sigma_{d}=\sigma_{\mathrm{AP}}-\sigma_{\mathrm{SP}}
$$

The correction of the electrical conductivity has allowed us to find its actual value. This allows to conclude that the chosen model and the applied extraction procedure are good.

In practice, we do not have material without losses and cannot perform dielectric measurements without losses.

From (9) we obtain

$$
\varepsilon_{r_{\mathrm{efff}, c}}=\frac{\varepsilon_{r_{\mathrm{efff}, m}} \cdot \ln \left(r_{0} / r_{i}\right)}{\ln (b / a)-\varepsilon_{r_{\mathrm{eff}, m}} \cdot \ln \left[b / a / r_{0} / r_{i}\right]} .
$$




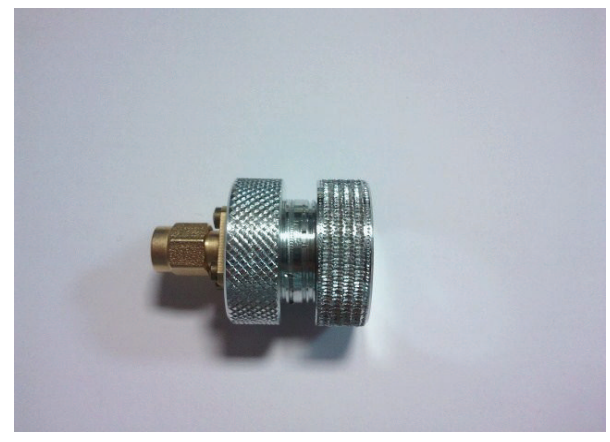

FIGURE 10: Photo of small coaxial line in reflection $(15 \mathrm{~mm})$.

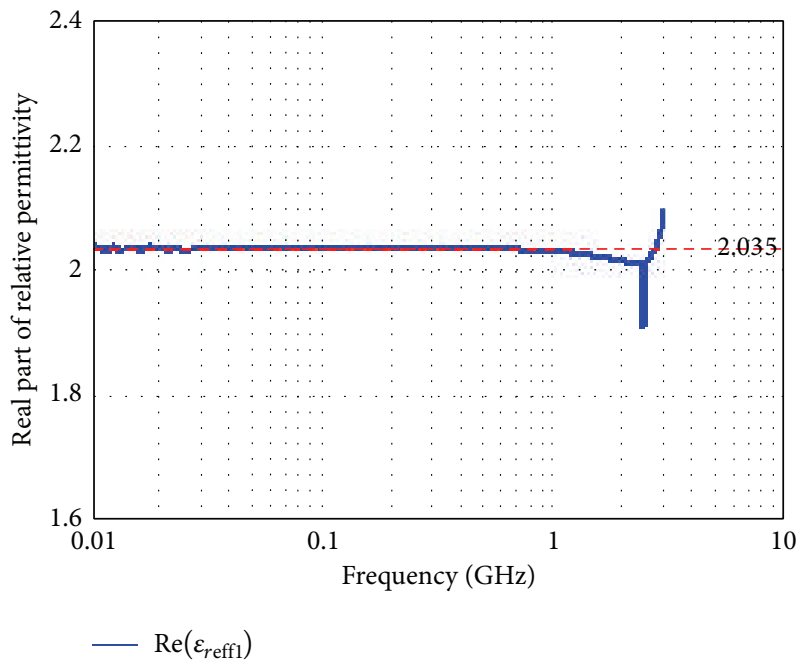

Figure 11: Teflon real relative permittivity (sample of $15 \mathrm{~mm}$ ).

To correct error of eccentricity we add the following formula:

$$
\varepsilon_{r_{\mathrm{eff}}}=\frac{\varepsilon_{r_{\mathrm{eff}}} c}{\sqrt{1+\left(\left(S \cdot \Delta \cdot \varepsilon_{r_{\mathrm{eff}}} c\right) / \alpha\right)^{2}}}
$$

where $\alpha=\log (b / a), \Delta=2(d-a) /(d+a), \Delta$ is a deviation fraction on inner diameter, $d$ is the air gap between central conductor and sample, and $s=\Delta c /(d-a)$ is the parameter of eccentricity.

Without eccentricity $(s=0)$ and with complete eccentricity $(s=1)$.

\section{Simulation Results}

The simulation results of the set of Figures 1 to 8 were obtained after extraction of the permittivity from the $S 11$ parameters. In Figures 3 and 4, we can see that the real part of the relative permittivity (of Delrin) and the dielectric losses decrease when the frequency and the value of the gaps increased, whereas Figure 5 shows that the error of relative permittivity of Delrin increases with the value of the gaps and weak for a line without gap. After correction, the results of Figures

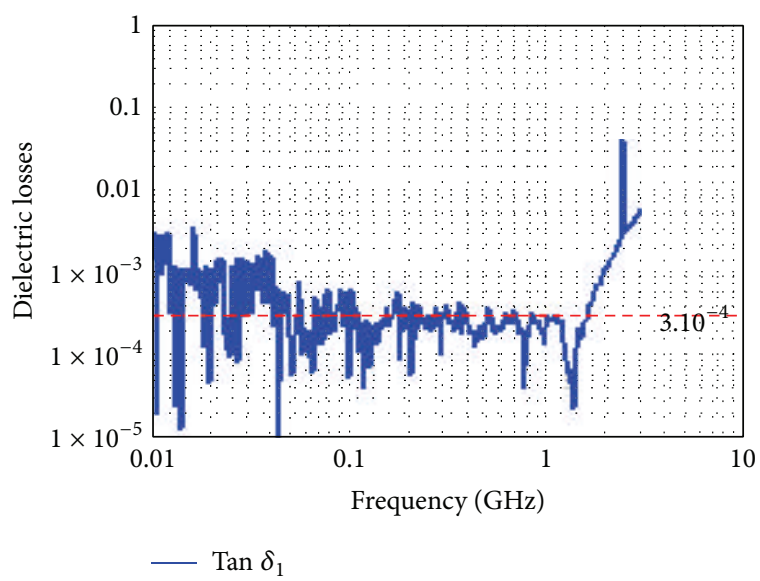

FIGURE 12: Teflon dielectric losses (sample of $15 \mathrm{~mm}$ ).

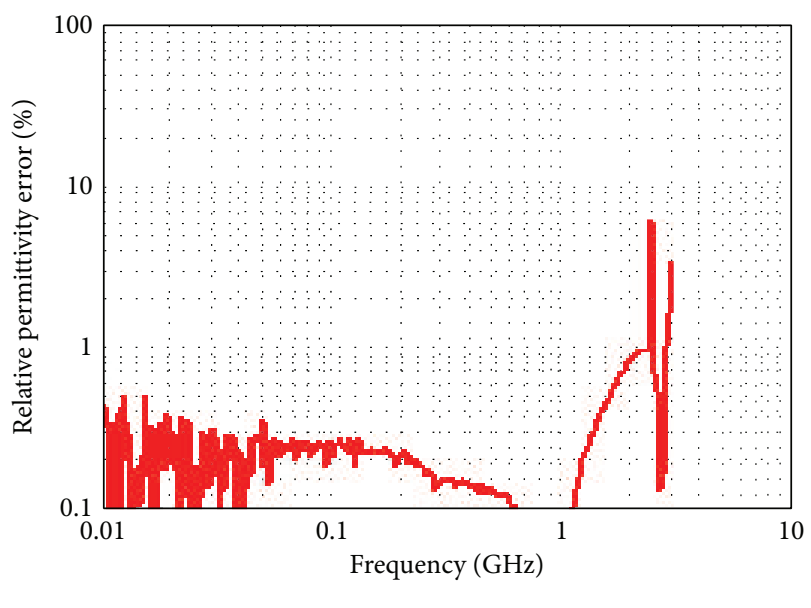

— Err_Re $\varepsilon_{r}$

FIGURE 13: Teflon relative permittivity error (sample of $15 \mathrm{~mm}$ ).

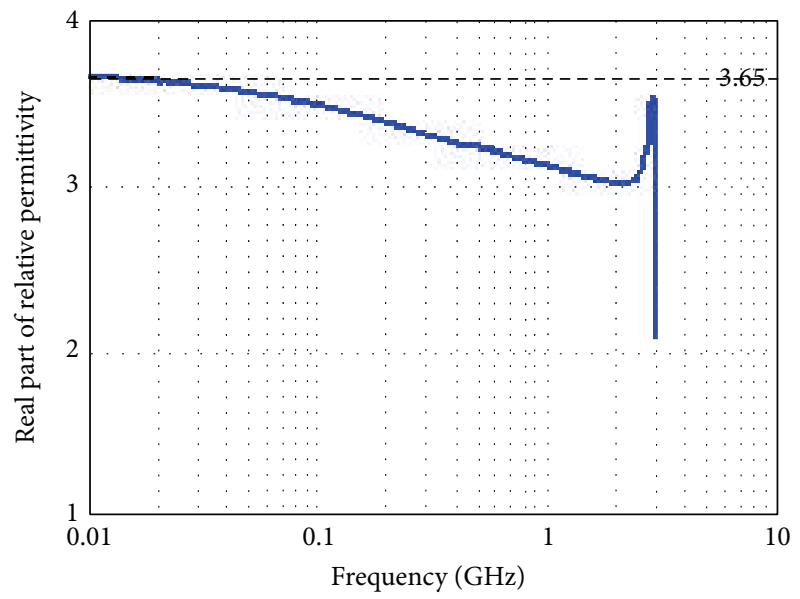

FIGURE 14: Delrin real part of effective relative permittivity (sample of $15 \mathrm{~mm}$ ). 


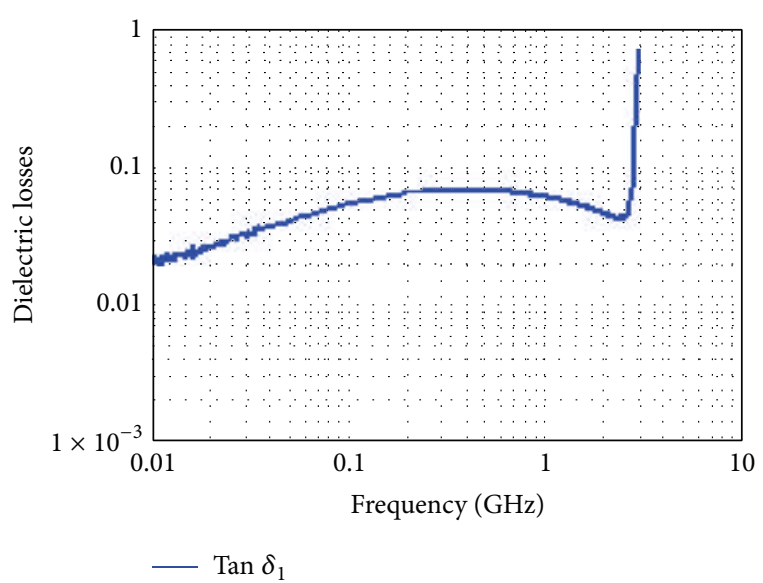

FIGURE 15: Delrin dielectric losses (sample of $15 \mathrm{~mm}$ ).

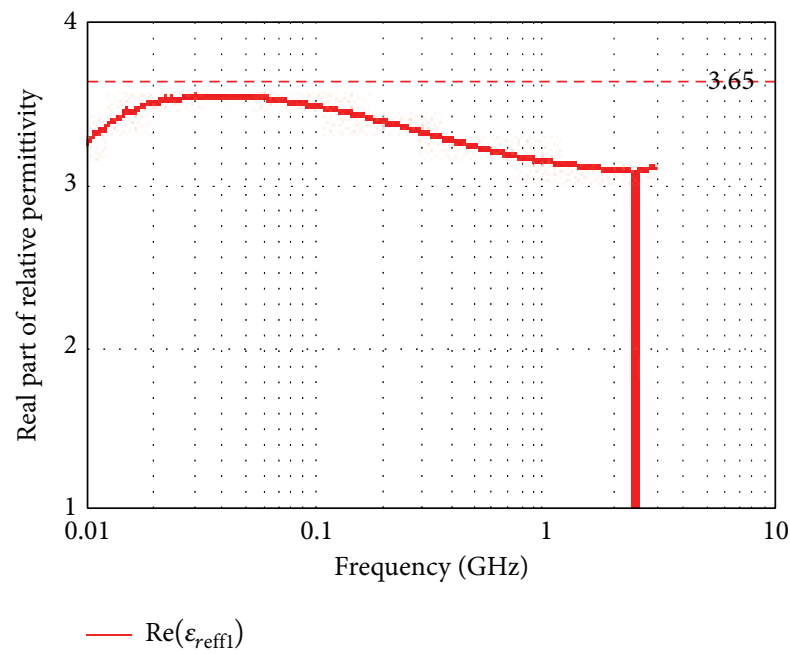

FIGURE 16: Delrin relative permittivity (sample of $5 \mathrm{~mm}$ long and with no air gap).

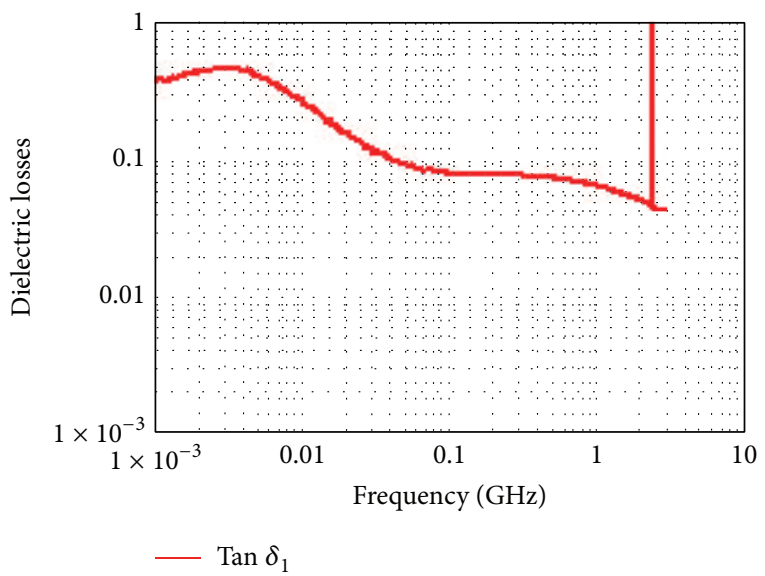

FIGURE 17: Delrin dielectric losses (sample of $5 \mathrm{~mm}$ long and with no air gap).

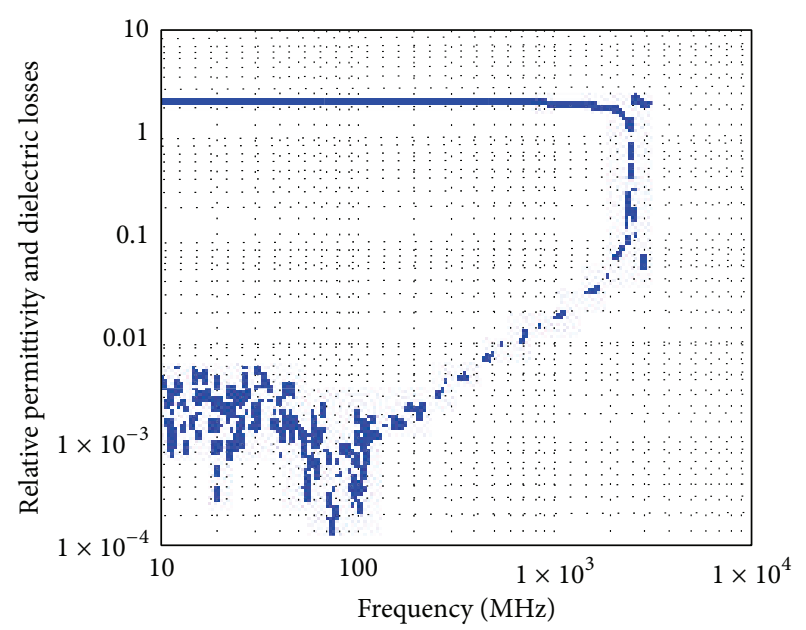

$$
\begin{aligned}
& \ldots \operatorname{Re}\left(\varepsilon_{r \text { ref }, m}\right) \\
& \ldots=\operatorname{Tan} \delta_{m}
\end{aligned}
$$

FIGURE 18: Teflon relative permittivity and dielectric losses (sample of $5 \mathrm{~mm}$ long and with no air gap).

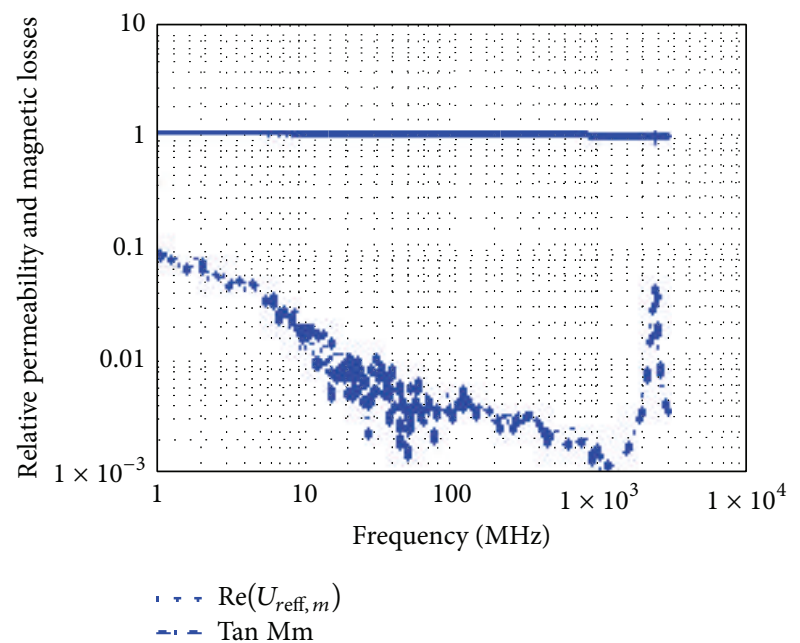

FIGURE 19: Teflon relative permeability and magnetic losses (sample of $5 \mathrm{~mm}$ long and with no air gap).

6,7 , and 8 show clearly the improvement of the results and the cancellation of the effect of the air gap. We see that the three curves merge into one, especially for the dielectric losses.

\section{Experimental Validation}

Before beginning measurements, we must define the reference plans that will support the practical implementation of the cell, and then we perform the calibration procedure to eliminate systematic errors of the measurement of 
the reflection coefficient. Finally, we validate the implementation method from the measurement of the complex permittivity of dielectric materials with well-known properties.

6.1. Realization of the Measuring Cell. To ensure the representativeness of the measurements performed on the discontinuity line/guide with respect to the electromagnetic properties of the samples, the measuring cell must have an outside diameter equal to $11.5 \mathrm{~mm}$. Under these conditions, the connection of the measuring cell to the vector network analyser requires the use of a coaxial/coax transition. This transition consist in an SMA connector, suitable for the connecting cable of the network analyser (diameter $3.5 \mathrm{~mm}$ ) and a coaxial conical portion which ensures the progressive variation of the diameter of the line between the coaxial line and the discontinuity, which has a line diameter of $5 \mathrm{~mm}$ (Figure 9).

To limit the effets of propagation discontinuity in the conical section, the ratio between the internal diameter of the outer conductor and the diameter of the central conductor is, on the whole length of the section, $2.3 \mathrm{~mm}$ (conditions of 50 ohms impedance matching).

6.2. Experimental Results. To exploit reflectance measures, we used a network analyser type VNA.E5062A. This implies positioning the reference plan of the measurement of the reflection coefficient at the end of the coaxial access line on the front face of the cylindrical sample characterization (Figure 10).

The experimental results of Figures 11, 12, and 13 present, respectively, the relative permittivity, the dielectric losses, and the relative permittivity error for Teflon (sample of $15 \mathrm{~mm}$ ). In Figures 14, 15, 16, 17, and 18 we plot the parameters of Delrin. These results were considered very good since the obtained values of all these parameters were very near to the typical values in this considered frequency range. Figures 17 and 18 are obtained for Derlin without air gap (sample of $5 \mathrm{~mm}$ ). We can notice, from Figure 18, that the losses are less than 0.1 after $50 \mathrm{MHz}$. For the same sample and in the same conditions, the simulation and experimental results are very close. This good agreement proves, for the corrected measurements by using the kit short-circuit/open-circuit/standard material, that the used technique and the calibration procedure were successful over the entire frequency band.

In Figures 18 and 19, we plot, respectively, the relative permittivity and the relative permeability and magnetic losses of Teflon (sample of $5 \mathrm{~mm}$ long and with no air gap). These figures show that the losses are constant over the entire frequency range. Figure 20 illustrates the relative permittivity for Delrin (sample of $5 \mathrm{~mm}$ long and with air gap of $0.5 \mathrm{~mm}$ in inner diameter). The red traces are the corrected values, obtained by using (11) and (12) to take into account the air gap effects.

We present, respectively, in Figure 21, the initial and corrected values of relative permittivity for Teflon (sample of $5 \mathrm{~mm}$ long and with air gap of $0.5 \mathrm{~mm}$ in inner diameter). We

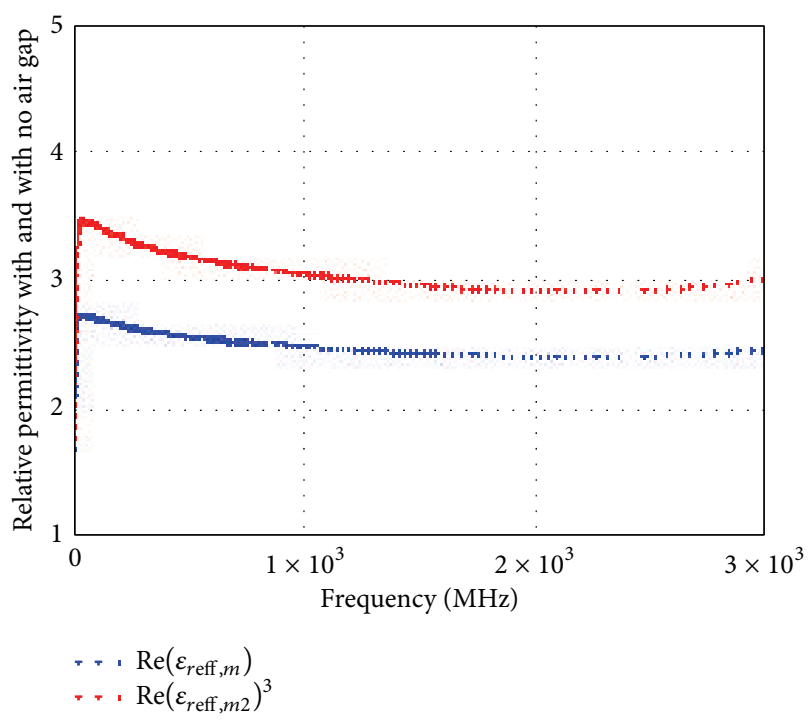

FIGURE 20: Delrin relative permittivity (sample of $5 \mathrm{~mm}$ long and with air gap of $0.5 \mathrm{~mm}$ in inner diameter).

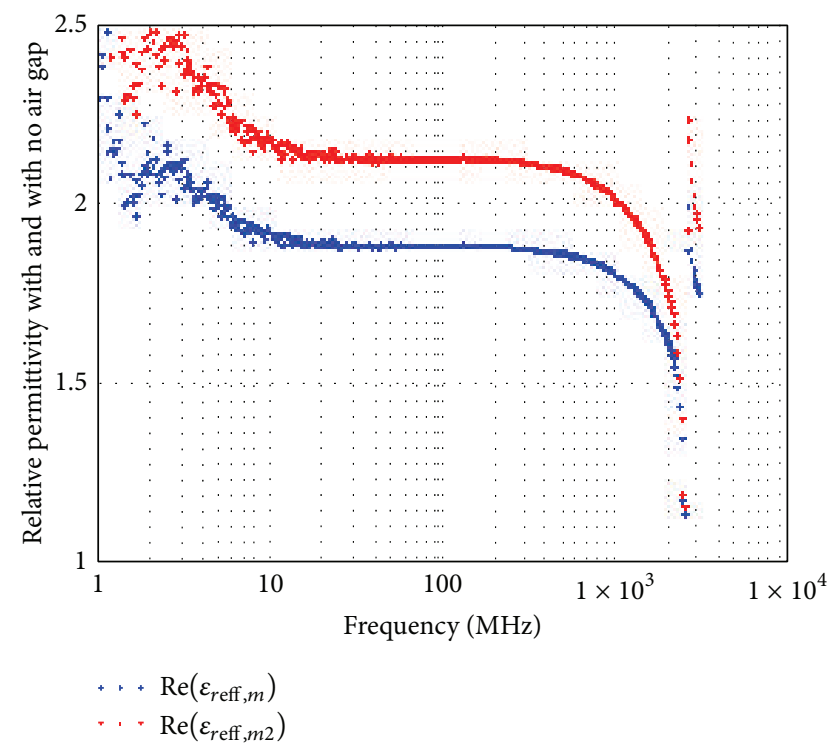

Figure 21: Teflon relative permittivity (sample of $5 \mathrm{~mm}$ long and with air gap of $0.5 \mathrm{~mm}$ in inner diameter).

notice here that, after correction, the relative permittivity is improved.

\section{Application of the Method for Multilayer Materials}

We will show that the proposed method can also be applied, successfully, to multilayer materials. We rely primarily on the principles of the coaxial probe in reflection. Let us put 
a bilayer dielectric in the metallic cavity (Figure 22(a)). In Figure 2 we invert the position of the layers. In literature, studies have shown that the coaxial probe is sensitive to the surface of the sample which is in contact with the central conductor [6]. This sensitivity is due to the distribution of electromagnetic fields in the vicinity of the central conductor $[7,8]$. This implies that the overall capacitance created is not the same as the orientation of the sample $\left(\varepsilon_{r 1} \neq \varepsilon_{r 2}\right)$.

7.1. Extraction of Each Layer's Complex Permittivity. We consider that the bilayer dielectric in contact with the greatest metal surface (point C) entails that overall losses are depending on the orientation of the sample. Moreover, at the interface of the two dielectrics (point B), the capacitance is the same regardless of the orientation of the sample.

We know that

$$
\begin{gathered}
\gamma l=\frac{j w l \sqrt{\varepsilon_{r}}}{c}, \\
\gamma_{\text {tot }} l_{\text {tot }}=\gamma_{1} l_{1}+\gamma_{2} l_{2}, \\
l_{\text {tot }}=l_{1}+l_{2} .
\end{gathered}
$$

When we put (13) in (14) we obtain the effective permittivity of the first layer as a function of that of the reference:

$$
\varepsilon_{r \text {-Delrin }}=\left(2 \cdot \sqrt{\varepsilon_{r \text {-tot }}}-\sqrt{\varepsilon_{r \text {-Teflon }}}\right)^{2} .
$$

This extraction method has the privilege of not necessarily being based on prior knowledge of the thickness of each layer. Only the thickness of the aggregate layer must be known in order to meet the dimensions of the test frame.

7.2. Experimental Results. We have plotted in Figures 23 and 24 , respectively, the relative permittivity and the magnetic losses of Delrin (sample $5 \mathrm{~mm}$ without air gap). In Figure 23 we show that the read plot which represents the relative permittivity of the Delrin, obtained using the bilayers methods, is pratically the same blue plot obtained with single sample, in the same frequency range between $10 \mathrm{MHz}$ and $1 \mathrm{GHz}$ but the accepted value is until $100 \mathrm{MHz}$ as shown in Figure 24; in this range of frequecy the dielectric losses are between $2 \%$ at $10 \mathrm{MHz}$ and $5 \%$ at $100 \mathrm{MHz}$. We have obtained a good precision.

The use of coaxial fixture in reflection so as to characterize multilayers materials has led to satisfactory results. The application of dielectric Delrin permits us to prove the method's feasibility.

Meanwhile, the method is based on the principle that electrical parameters of one layer are well known and that the global thickness of the layers is precisely known.

\section{Conclusion}

We presented the technique of coaxial probe having a cavity end to characterize single and bilayer materials. Its feasibility was validated by electromagnetic simulation results and experimental results after completing the experimental aluminum test frame which is lighter than copper.

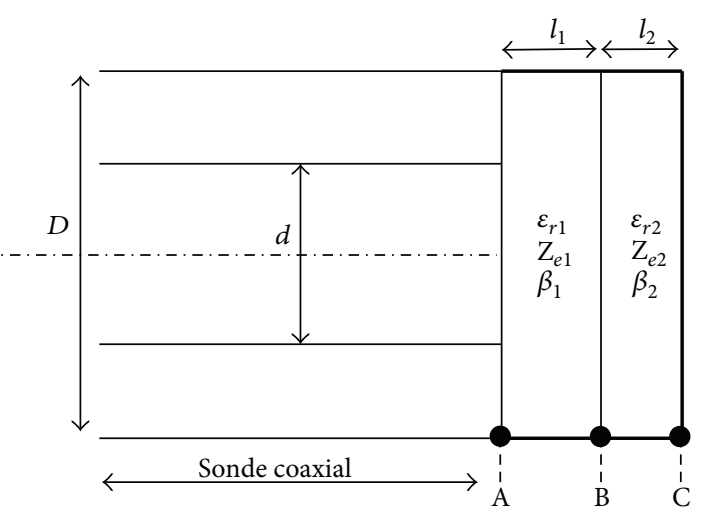

(a)

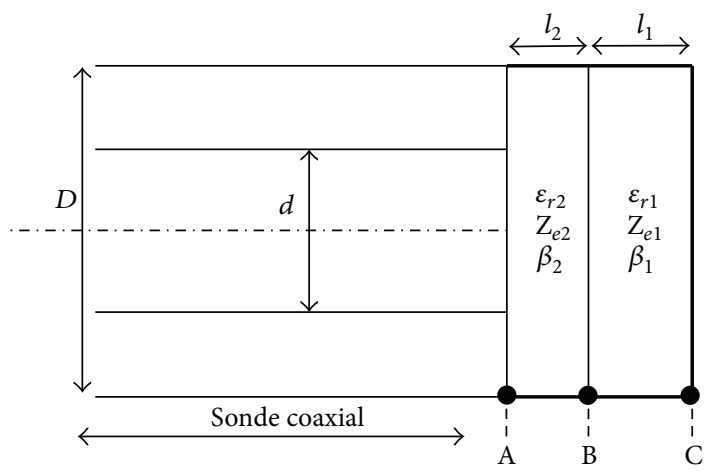

(b)

FIgURE 22: (a) Bilayer coaxial probe in the presence of a sample of the same thickness (configuration 1). (b) Bilayer coaxial probe in the presence of a sample of the same thickness (configuration 2).

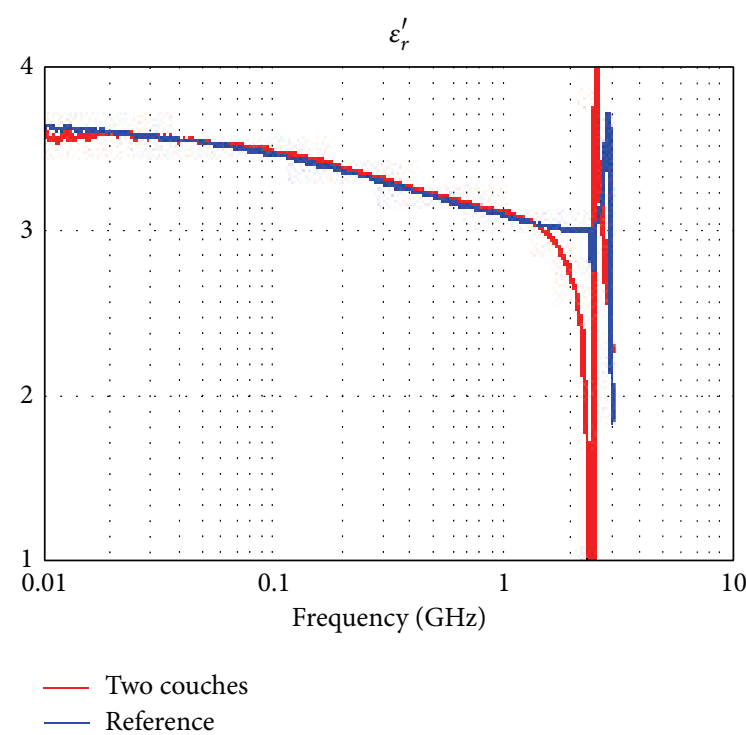

FIGURE 23: Delrin relative permittivity (sample of $5 \mathrm{~mm}$ long and with no air gap). 


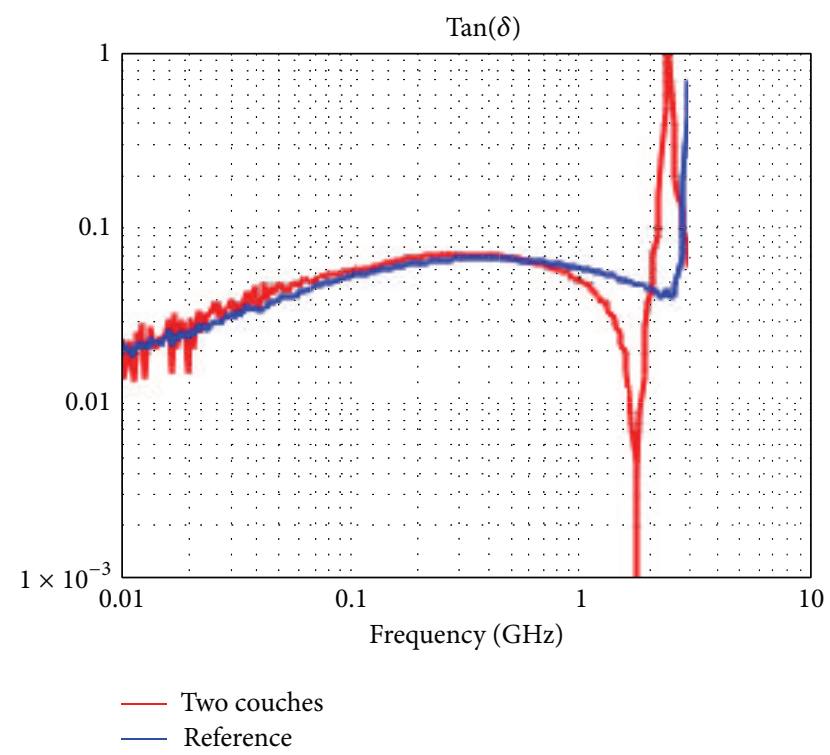

FIGURE 24: Delrin dielectric losses (sample of $5 \mathrm{~mm}$ long and with no air gap).

This technique is applicable to dielectrics and semiconductors. It is solely based on the reflection parameters using a capacitive model and helps to increase frequency band of model. Although efforts are yet to be made for its improvements, especially in the extraction losses for multilayer materials, this method is easy to implement and rapid in extracting electrical parameters and precise in terms of repeatability whose relative error is about $5 \%$. This method offers the possibility to extract low loss tangent $\left(2 \cdot 10^{-4}\right)$ up to $1 \mathrm{GHz}$ using an SMA connector.

\section{Conflict of Interests}

The authors declare that there is no conflict of interests regarding the publication of this paper.

\section{References}

[1] C. C. Courtney, "Time-domain measurement of the electromagnetic properties of materials," IEEE Transactions on Microwave Theory and Techniques, vol. 46, no. 5, pp. 517-522, 1998.

[2] T. W. Athey, M. A. Stuchly, and S. S. Stuchly, "Measurement of radio frequency permittivity of biological tissues with an openended coaxial line: part I," IEEE Transactions on Microwave Theory and Techniques, vol. 30, no. 1, pp. 82-86, 1982.

[3] K. J. Bois, L. F. Handjojo, A. D. Benally, K. Mubarak, and R. Zoughi, "Dielectric plug-loaded two-port transmission line measurement technique for dielectric property characterization of granular and liquid materials," IEEE Transactions on Instrumentation \& Measurement, vol. 48, no. 6, pp. 1141-1148, 1999.

[4] S.-G. Pan, "Characteristic impedances of coaxial system consisting of circular and noncircular conductors," IEEE Transactions on Microwave Theory and Techniques, vol. 36, no. 5, pp. 917-921, 1988.
[5] J. Baker-Jarvis, Transmission/Reflection and Short-Circuit Line Permittivity Measurements, National Institute of Standards and Technology, 1990.

[6] M. Moukanda, F. Ndagijimana, J. Chilo, and P. Saguet, "Caractérisation des Matériaux Multicouches en Utilisant une Sonde Coaxiale en Présence d'un Plan De Masse," in 15ème Journées Nationales Micro-Ondes (J.N.M), Toulouse, France, Mai 2007.

[7] N. Belhadj-Tahar, O. Meyer, and A. Fourrier-Lamer, "Broadband microwave characterization of bilayered materials using a coaxial discontinuity with applications for thin conductive films for microelectronics and material in air-tight cell," IEEE Transactions on Microwave Theory and Techniques, vol. 45, no. 2, pp. 260-267, 1997.

[8] N. Belhadj-Tahar, O. Dubrunfaut, and A. Fourrier-Lamer, "Broad-band microwave characterization of a tri-layer structure using a coaxial discontinuity with applications for magnetic liquids and films," IEEE Transactions on Microwave Theory and Techniques, vol. 46, no. 12, pp. 2109-2116, 1998. 

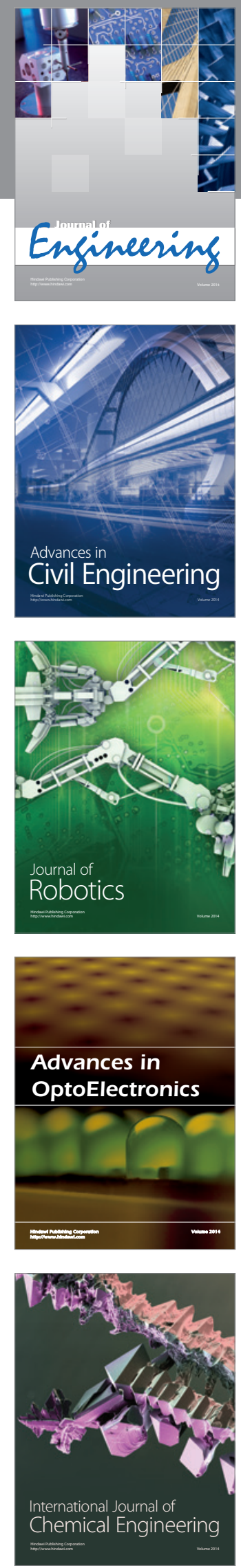

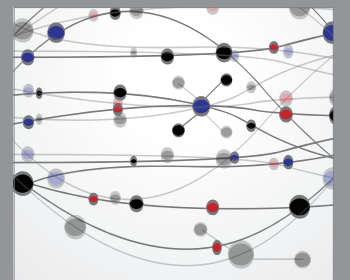

The Scientific World Journal
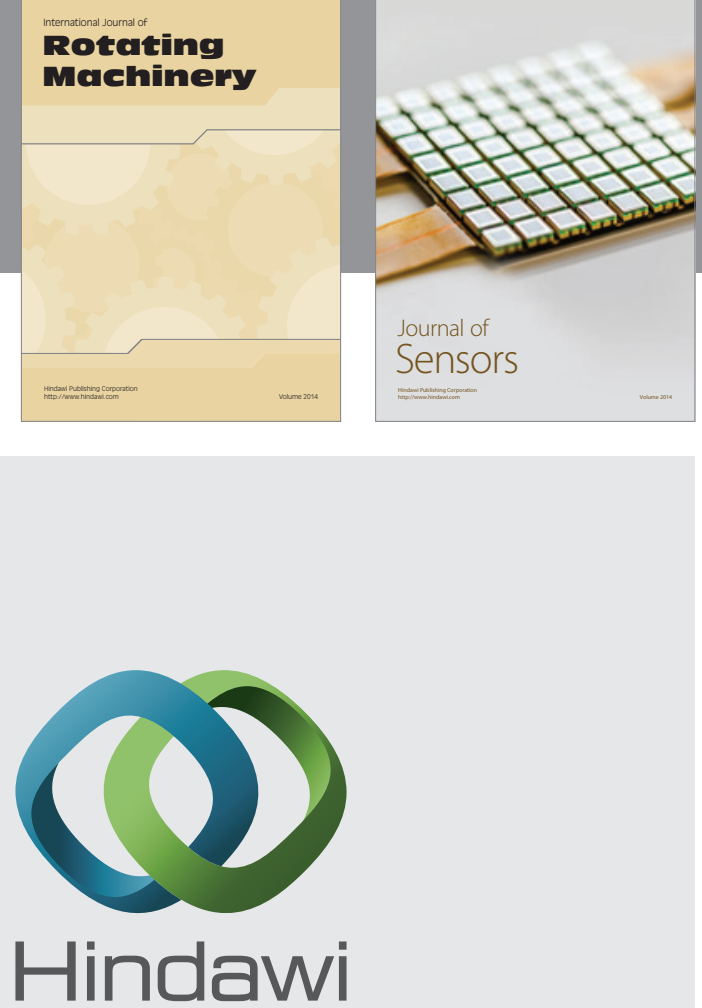

Submit your manuscripts at http://www.hindawi.com
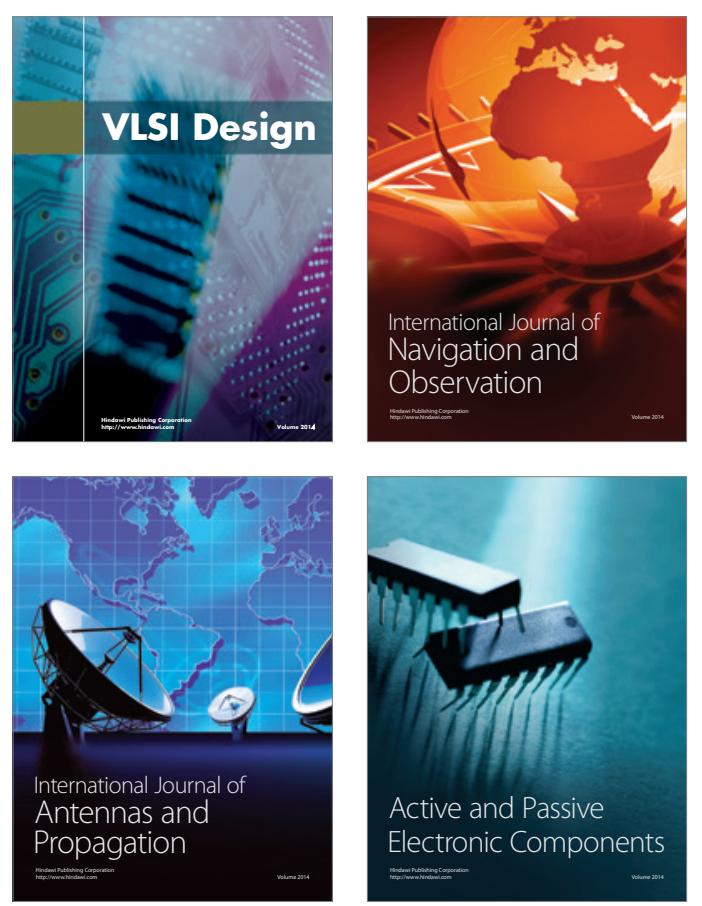
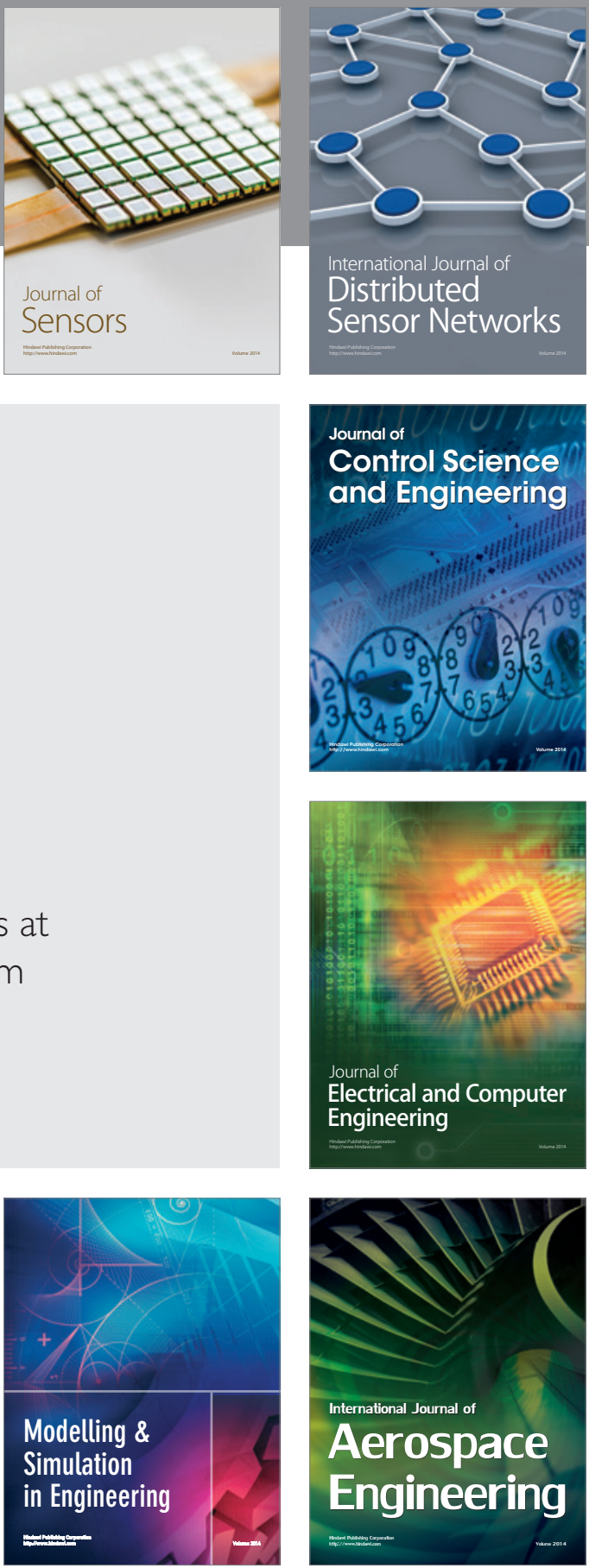

Journal of

Control Science

and Engineering
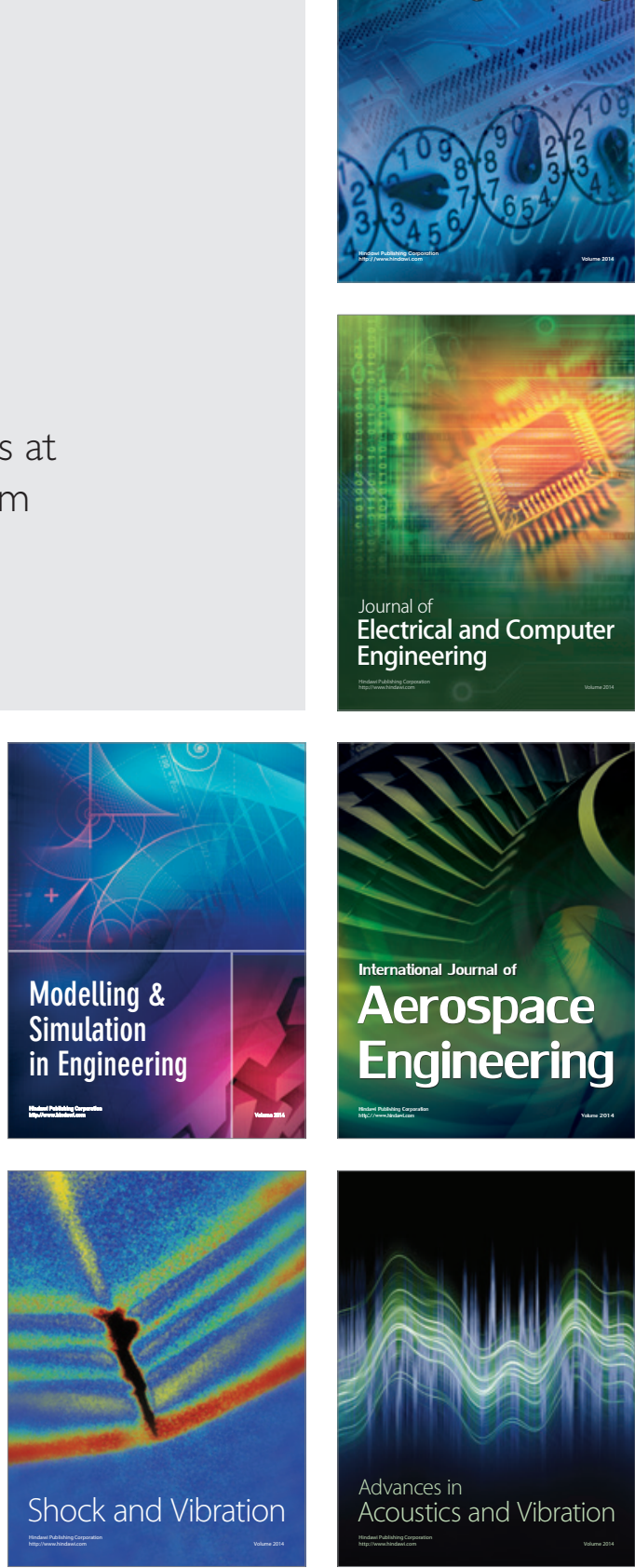